\title{
Heron-mouth neuroendoscopic sheath-assisted neuroendoscopy plays critical roles in treating hypertensive intraventricular hemorrhage
}

\author{
Jie Xu, Siyuan Ma, Weijiang Wu, Wenfeng Fang, Aihua Zhu, Chun Ge, Hua Lu \\ Department of Neurosurgery, Affiliated Hospital of Jiangnan University (Wuxi Third People's Hospital), Wuxi, China
}

Videosurgery Miniinv 2021; 16 (1): 199-210

DOI: https://doi.org/10.5114/wiitm.2020.99351

\begin{abstract}
Introduction: Neuroendoscopy is widely applied for treating hypertensive intracerebral hemorrhage.

Aim: To explore the effects of heron-mouth neuroendoscopic sheath-assisted neuroendoscopy on treatment of hypertensive intraventricular hemorrhage.

Material and methods: A type of heron-mouth neuroendoscopic sheath combining the advantages of minimally invasive columnar endoscopic sheath and open operation methods was designed. The end of sheath catheter could be dilated if necessary, without increasing risk of cortex injury. Heron-mouth neuroendoscopic sheath-assisted neuroendoscopy was applied in treatment of hypertensive intraventricular hemorrhage. A total of 19 patients with hypertensive intraventricular hemorrhage were selected and divided into an external ventricular drainage + urokinase group and a neuroendoscopy group. Hematoma clearance rate, surgical time, ventricular drainage time, intracranial infection, hydrocephalus and Glasgow Outcome Score (GOS) at 3 months after the operation were compared between two groups. Results: Hematoma clearance rate, ventricular drainage time, mortality rate and GOS at 3 months after surgery in the neuroendoscopy group were significantly better compared to those in the external ventricular drainage + urokinase group $(p<0.05)$. Postoperative complications, including intracranial infection hydrocephalus and pulmonary infection in the neuroendoscopy group, were less numerous compared to those in the external ventricular drainage + urokinase group, but without statistical significance $(p>0.05)$. However, surgical time was significantly longer in the neuroendoscopy group compared to that in the external ventricular drainage + urokinase group $(p<0.05)$. There was no significant difference in incidence rate of hydrocephalus between the two groups $(p>0.05)$.

Conclusions: Clinical effects of heron-mouth neuroendoscopic sheath-assisted neuroendoscopy were better than those of external ventricular drainage combining urokinase dissolution in treating hypertensive intraventricular hemorrhage.
\end{abstract}

Key words: heron-mouth neuroendoscopic sheath, neuroendoscopy, hypertensive intraventricular hemorrhage, hematoma.

\section{Introduction}

The application of neuroendoscopy in operative treatment of hypertensive intracerebral hemorrhage has gradually become a hotspot among neurosurgeons in the whole world [1]. Neuroendoscopy is characterized by minimal invasion, sufficient light and multiple angles of view, demonstrating unique advantages in treating hypertensive intraventricular hemorrhage [2-4]. Auer et al. [5] firstly reported endoscope-assisted evacuation of intracerebral hema-

\section{Address for correspondence}

Hua Lu MD, Department of Neurosurgery, Affiliated Hospital of Jiangnan University (Wuxi Third People's Hospital), Wuxi, China,

e-mail: luhua1969hot@163.com 
toma, with a surgery-related mortality rate of $30 \%$. The effect of the operation on patients with cortical hemorrhage is better than that of conservative treatment [5]. Meanwhile, there are no significant differences for prognosis of thalamic and putamen hemorrhage between surgery and conservative treatment. With the wide and skilled application of endoscopy in neurosurgery, endoscopy has demonstrated significant effects in treating various types of cerebral hemorrhage. According to a large number of papers [6-9], the average clearance rate of hematoma using neuroendoscopy ranges from $79.2 \%$ to $95.0 \%$, and the long-term prognosis of patients is also favorable. Basaldella et al. [10] reported that the therapeutic effect of neuroendoscopic evacuation of hematoma is better than that of external ventricular drainage alone in patients with moderate-severe ventricular hemorrhage. The neuroendoscopic evacuation of hematoma has demonstrated definite efficacy. Moreover, with the innovation and improvement of neuroendoscopic instruments, neuroendoscopy would develop more advantages in treatment of hypertensive intracerebral hemorrhage [11].

In recent years, endoscopic instruments and accessories have been designed and innovated by researchers in China and foreign countries [12, 13]. A type of heron-mouth neuroendoscopic sheath catheter with the minimally invasive advantage of endoscopic surgery has been designed by our department previously [1].

In this study, the sheath catheter was applied in neuroendoscopy in treatment of 10 cases of hypertensive intraventricular hemorrhage. Meanwhile, another 9 patients with hypertensive intraventricular hemorrhage were selected and administered with traditional external drainage combining urokinase. Then, the differences between the above two methods were compared, and satisfactory effects were obtained.

\section{Aim}

This study aimed to explore the effects of heron-mouth neuroendoscopic sheath-assisted neuroendoscopy on treatment of hypertensive intraventricular hemorrhage.

\section{Material and methods}

\section{Case selection}

The present study prospectively selected a total of 19 patients with hypertensive intraventricu- lar hemorrhage. The inclusion criteria were as follows: 1) Patients aged from 18 years to 80 years demonstrating known symptom onset within $24 \mathrm{~h}$ of initial computed tomography (CT) scan verifying intraventricular hemorrhage. 2) Both diastolic pressure and systolic pressure were higher than normal. 3) Assignment for each group within $72 \mathrm{~h}$ after the CT scan diagnosis for hypertensive intraventricular hemorrhage. The exclusion criteria were as follows: 1) Patient with pregnancy. 2) Ongoing bleeding or coagulopathy. 3) Superficial or multifocal bleeding appears at the vascular puncture or access sites or the sites with the surgical intervention. 4) Other conditions which might bring a risk to the patients if the used operation was conducted.

In the neuroendoscopy group, there were 7 males and 3 females aged from 54 years to 61 years (with an average age of 58.6 years). The preoperative Glasgow coma score (GCS) was 4-8 points in 5 cases and 9-12 points in 5 cases. In the external ventricular drainage + urokinase group, there were 5 males and 4 females aged from 48 years to 65 years, with an average age of 60.7 years. The preoperative GCS was $4-8$ points in 5 cases and 9-12 points in 4 cases. There were no statistically significant differences between the two groups according to the equilibrium test.

\section{Operation methods}

Neuroendoscopy group: The operation was performed in 5 cases under general anesthesia (4 cases under local anesthesia) through the approach of the anterior horn of the lateral ventricle anterior to the coronal suture. A $3 \mathrm{~cm}$-long skin incision was made at $2 \mathrm{~cm}$ in front of the coronal suture and $2.5 \mathrm{~cm}$ near the midline. Then, the skull was drilled and expanded until the bone window with $2 \mathrm{~cm}$ in diameter. The dura mater was cut in a cross shape and suspended, and the blood vessels on the surface of the cortex were electro-coagulated. Then, the heron-mouth neuroendoscopic sheath (Photo 1) was placed along the anterior horn of the lateral ventricle, and the sheath catheter was dilated and fixed with the self-made fixing device. The endoscope was pushed forward accompanied by continuous washing with normal saline at $36-37^{\circ} \mathrm{C}$. The blood clots in the anterior horn of the lateral ventricle and somatic part were removed, and the intraventricular hematoma was cleaned up. The ventricle was 

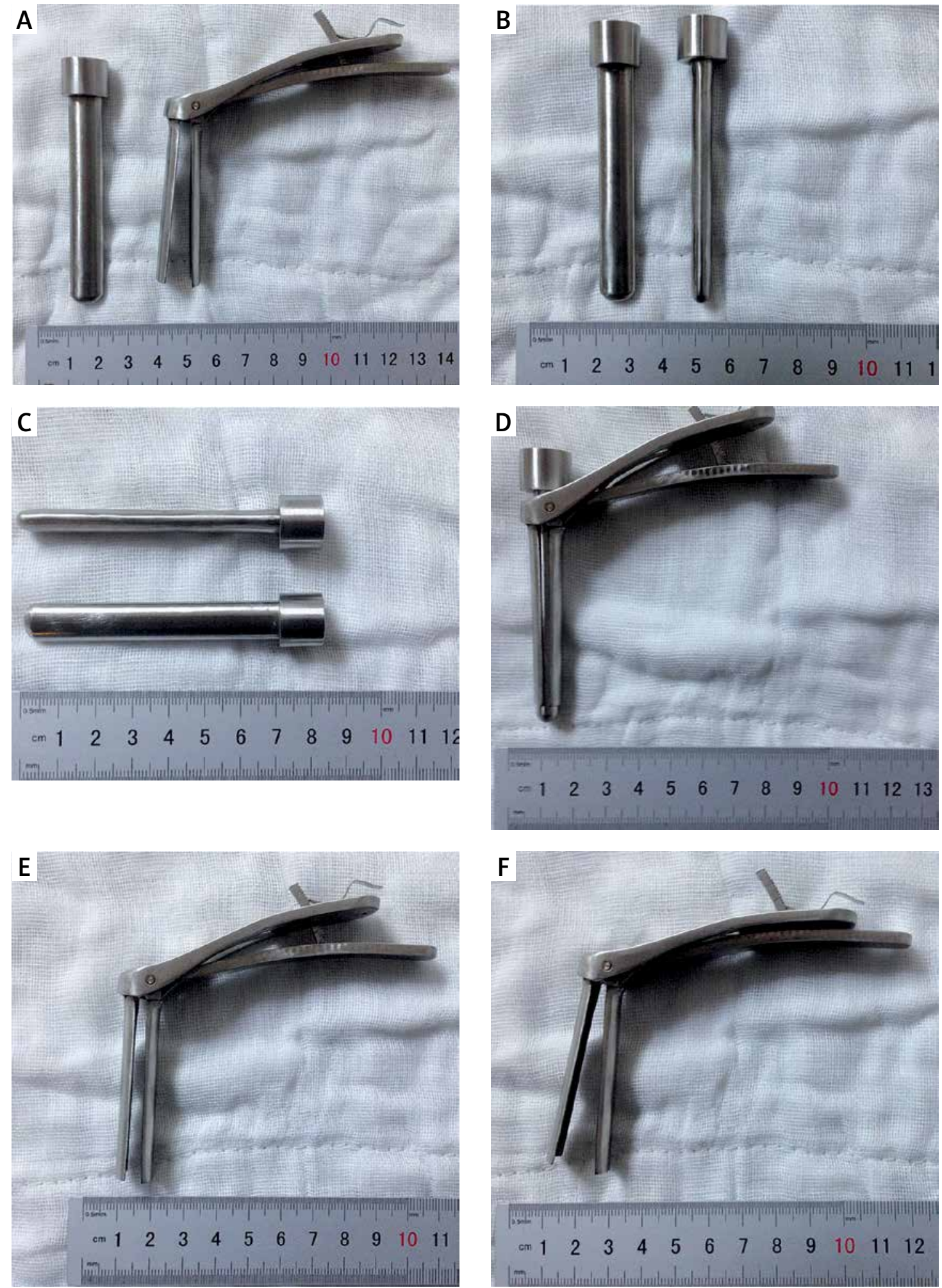

Photo 1. Real picture of heron-mouth neuroendoscopic sheath. A - Sheath body and sheath core. B, C - Diameter and length of the oval sheath core. D - Sheath core was placed into sheath body when the puncture was conducted. E - Sheath core was removed after the puncture. F - The sheath catheter was expanded with the sheath handle 

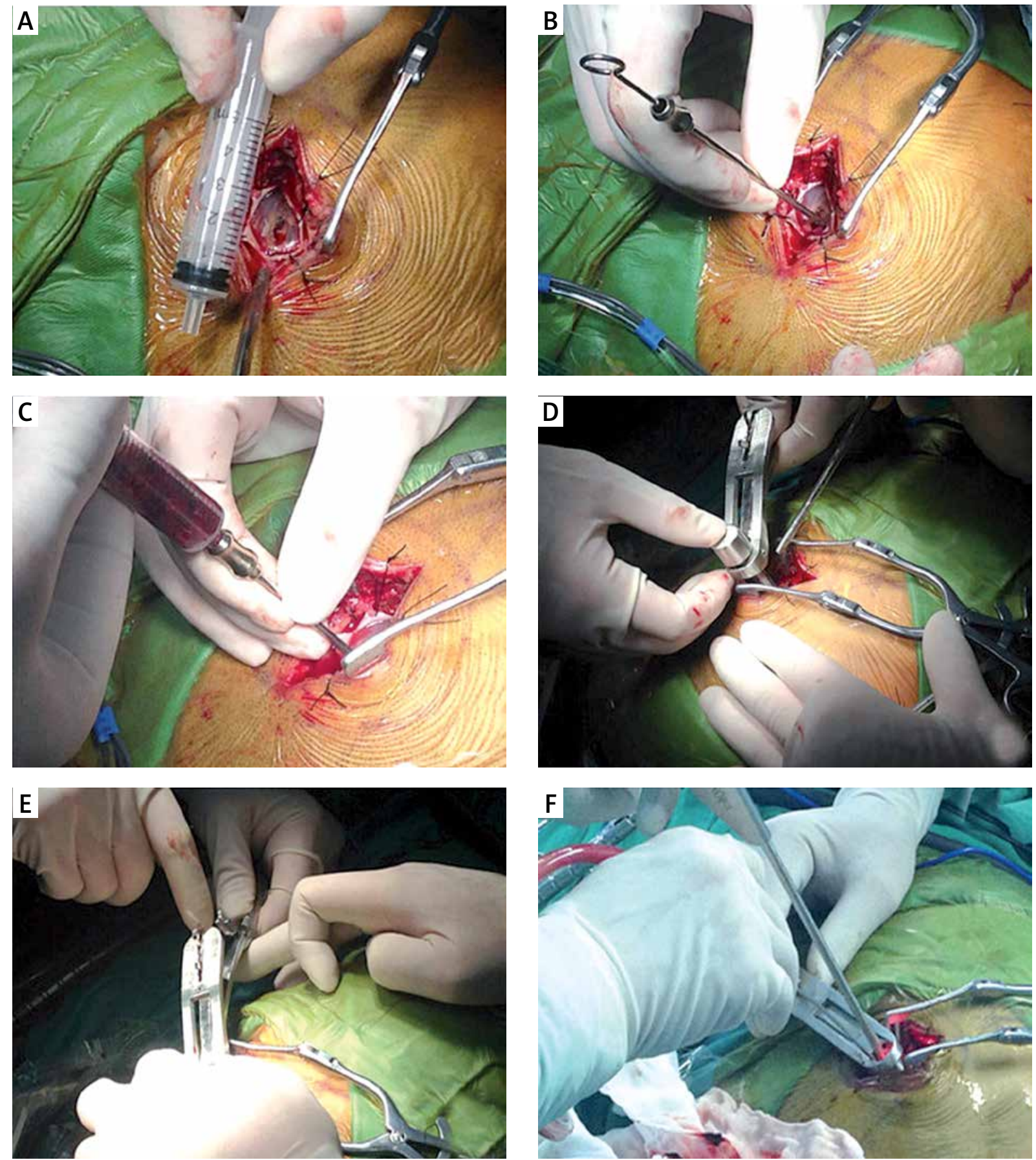

Photo 2. Diagrams of Heron-mouth neuroendoscopic sheath-assisted neuroendoscopy of intraoperation. A - Performing a 3-4 cm straight incision, drilling and enlarging the bone window with a diameter of $1.5-2.5 \mathrm{~cm}$ to incise the dura mater. B - Suspending the dura mater and inserting the needle slowly to avoid the blood vessels on the surface of the cerebral cortex. $\mathbf{C}$ - Detecting the hematoma and releasing some hemorrhagic fluid for decompression. D - Expanding the cortical fistula and inserting it slowly into the egret-beak neuroendoscope sheath. E - Snaking traction and egret-beak intra. F - Pulling out the sheath core, dilating the sheath, opening the hematoma cavity, and removing the hematoma under endoscope with help of sheath tube 
washed with normal saline to expose the interventricular foramen, and the hematoma in the interventricular foramen was gently sucked out. For patients with a third ventricular hematoma, the endoscope was pushed forward and the blood clots in the third ventricle were removed. For patients with bilateral ventricular cast hematoma, when the septum pellucidum had no blood vessels it was opened. Meanwhile, the contralateral ventricular hematoma was gently sucked out through the septum pellucidum. Then, the external ventricular drainage tube was placed under direct vision, the sheath catheter was closed and the sheath was withdrawn. The wound was sutured layer by layer. After surgery, the external ventricular drainage tube was removed according to the postoperative CT results. The tube was clamped for $24 \mathrm{~h}$ before withdrawal. The processes for heron-mouth neuroendoscopic sheath-assisted neuroendoscopy of intraoperation are illustrated in Photo 2.

External ventricular drainage + urokinase group: A $3 \mathrm{~cm}$-long longitudinal incision was made at $2 \mathrm{~cm}$ in front of the coronal suture and $2.5-3.0 \mathrm{~cm}$ near the midline in a supine position under local anesthesia. The skin was retracted with the mastoid retractor, the skull was drilled and the dura mater was cut in a cross shape. Then, the external ventricular drainage tube was placed and pushed forward parallel to the sagittal plane towards the connection of two external auditory canals. The inner core was withdrawn when there was an obvious sense of breakthrough or outflow of obsolete bloody fluid. Subsequently, the tube was pushed forward for another 2-3 cm after it was confirmed unobstructed, accompanied by continuous washing with normal saline. After that, the drainage tube was loosened at a low level and fixed, and the skin was sutured layer by layer, followed by postoperative CT dynamic observation. A total of 20 000-40 $000 \mathrm{U}$ of urokinase was dissolved in $2 \mathrm{ml}$ of normal saline and injected into the ventricle every day, and the tube was clamped for $2 \mathrm{~h}$ and loosened. The external ventricular drainage tube was removed according to the postoperative $\mathrm{CT}$ results, and the drainage tube was clamped for $24 \mathrm{~h}$ and kept open before withdrawal.

\section{Operative indications}

In this study, the operative indications for hypertensive intraventricular hemorrhage as follows:
1) intraventricular hemorrhage patients demonstrating increased intracranial pressure with brainstem compression signs, such as slow pulse, elevated blood pressure, slower respiratory rhythm and lower consciousness level; 2) patients illustrating the following signs: cerebellar hematoma volume more than $10 \mathrm{ml}$ or vermis more than $6 \mathrm{ml}$, hematoma breaking into the fourth ventricle or cistern and disappeared undergoing finger pressure, brainstem compression symptoms, acute and obstructive hydrocephalus symptoms; 3) severe ventricular hemorrhage of patients leading to the obstructive hydrocephalus.

For basal ganglionic hematoma, when the volume of hematoma in the basal ganglia was more than $30 \mathrm{ml}$, the neuroendoscopic surgery (open method) could be performed. For the thalamic hematoma, the limited local hematoma of the thalamus could be treated conservatively in advance, and external ventricular drainage + urokinase (conventional surgery) should be carried out if the hematoma condition gradually deteriorated. For the intracapsular hematoma of the thalamus, when the hematoma of the whole thalamus extended to the internal capsule or the external superior, both open and conventional surgery could be conducted. The amount of intraoperative bleeding was evaluated by an experienced surgeon using the visual method.

\section{Postoperative treatment}

All cases underwent the standard preoperative and postoperative treatment as well as electrocardiogram monitoring and intracranial pressure monitoring [14]. Sedative and analgesic treatment was performed; the antihypertensive drugs were pumped into the vein using the micro venous pump to control blood pressure at the normal status. A tracheostomy was performed to ensure good ventilation for patients with a long-term coma after the operation. The blood glucose was controlled and the water-electrolyte stability was maintained. In the late stage, according to our clinical experience, high pressure oxygen, acupuncture and physiotherapy could be performed to benefit the patients. The external hematoma drainage tube was generally removed within $48 \mathrm{~h}$ after the operation. The external ventricular drainage tube was removed according to postoperative CT results, and the tube was clamped for $24 \mathrm{~h}$ before withdrawal. 


\section{Observation indexes}

The postoperative residual hematoma, hematoma clearance rate, surgical time, amount of intraoperative bleeding and extubation time of the external ventricular drainage tube were observed. The GCS at 1 week after the operation and complications, such as infection and rebleeding, were also observed. The survival status was evaluated at 6 months after the operation mainly according to the Glasgow Outcome Score (GOS) and modified Rankin Scale (mRS) scoring criteria. Meanwhile, the cortex fistula in both groups was also determined and irrigated using saline until no obvious bleeding appeared (without a definite amount of irrigation fluid). The hemostasis was inspected under the endoscope, and then a drainage tube was placed.

\section{Ethics approval and consent to participate}

This study has been approved by the Ethical Committee of the Affiliated Hospital of Jiangnan University (Wuxi Third People's Hospital), Wuxi, China. Informed consent was obtained from their legally authorized representative persons or family members.

\section{Statistical analysis}

Data in this study were analyzed using professional IBM SPSS software v. 19.0 (IBM Corp.,
Armonk, NY, USA). The quantitative data (normal distribution) were represented as mean \pm standard deviation (SD) and analyzed using Student's $t$ test between two groups. The categorical data were presented as percentages and analyzed using the $\chi^{2}$ test between groups. Tukey's post hoc test validated analysis of variance (ANOVA) was used to compare data among multiple groups. A $p$-value less than 0.05 was considered to indicate a significant difference.

\section{Results}

Comparison for general data of patients with intraventricular hemorrhage between two groups

In order to guarantee accuracy for comparing parameters between the neuroendoscopy group and external ventricular drainage + urokinase group, general data of patients were compared. The results indicated that there were not significant differences for age, duration from onset to operation, GCS and Graeb intraventricular hemorrhage score, between the two groups (Table I, $t=0.425, t=0.864$, $t=0.166$ and $t=0.496$, respectively, $p=0.676$, $p=0.4, p=0.905$ and $p=0.661$, respectively). Therefore, it is comparable for parameters between the two groups.

Table I. Comparison of general data of patients with intraventricular hemorrhage

\begin{tabular}{|lcccc|}
\hline Group & Age [years old] & $\begin{array}{c}\text { Duration from onset } \\
\text { to operation }[\mathrm{h}]\end{array}$ & GCS & $\begin{array}{c}\text { Graeb intraventricular } \\
\text { hemorrhage score }\end{array}$ \\
\hline Neuroendoscopy group & $58.6 \pm 9.7$ & $7.3 \pm 2.2$ & $8.5(5-11)$ & $8.5(6-11)$ \\
\hline $\begin{array}{l}\text { external ventricular drain- } \\
\text { age + urokinase group }\end{array}$ & $60.7 \pm 11.6$ & $8.1 \pm 1.9$ & $8(5-11)$ & $8(6-11)$ \\
\hline$t$ & 0.425 & 0.864 & 0.166 & 0.496 \\
\hline$P$-value & 0.676 & 0.400 & 0.905 & 0.661 \\
\hline
\end{tabular}

Table II. Comparison of intraoperative data of cerebral hemorrhage in basal ganglia

\begin{tabular}{|lcccc|}
\hline Group & Surgical time [min] & $\begin{array}{c}\text { Amount of } \\
\text { intraoperative } \\
\text { bleeding [ml] }\end{array}$ & $\begin{array}{c}\text { Cortex fistula [mm] } \\
\text { [days] }\end{array}$ & $\begin{array}{c}\text { Extubation time } \\
\text { [days }\end{array}$ \\
\hline Neuroendoscopy group & $112.5 \pm 9.8$ & $101.0 \pm 12.0$ & 12 & $3.7 \pm 0.8$ \\
\hline $\begin{array}{l}\text { external ventricular drain- } \\
\text { age + urokinase group }\end{array}$ & $55.6 \pm 5.3$ & $84.4 \pm 15.1$ & 6 & $6.3 \pm 1.9$ \\
\hline$t$ & 15.998 & 2.629 & 19.436 & 6.856 \\
\hline$P$-value & $<0.001$ & 0.02 & $<0.001$ & $<.001$ \\
\hline
\end{tabular}




\section{Neuroendoscopy shortened extubation time}

The surgical time, amount of intraoperative bleeding, cortex fistula and extubation time were compared between the neuroendoscopy group and the external ventricular drainage + urokinase group. The results showed that neuroendoscopy treatment significantly shortened the extubation time compared to that in the external ventricular drainage + urokinase group (Table II, $t=6.856, p<0.001$ ). However, neuroendoscopy treatment prolonged surgical time (Table II, $t=15.998, p<0.001$ ), increased amount of intraoperative bleeding (Table II, $t=2.629$, $p=0.02$ ) and enlarged the cortex fistula (Table II, $t=19.436, p<0.001)$ compared to the external ventricular drainage + urokinase group.

\section{Neuroendoscopy enhanced hematoma clearance rate}

The hematoma clearance rate is a key index to evaluate efficacy of an operation. Therefore, it was tested in patients in both groups using CT assay (Photo 3) and endoscopic hematoma clearance assay (Photo 4). The results indicated that the hematoma clearance rate in the neuroendoscopy group was significantly higher compared to that in the external ventricular drainage + urokinase group (Table III, $\left.\chi^{2}=6.107, p=0.047\right)$.

\section{Neuroendoscopy only caused a lower post-operative complication rate}

In this study, the complications, including intracranial infection, hydrocephalus and pulmonary infection, were evaluated and compared between the neuroendoscopy group and the external ventricular drainage + urokinase group. The results indicated that complication rates of intracranial infection, hydrocephalus and pulmonary infection in the neuroendoscopy group were lower compared to those in the external ventricular drainage + urokinase group (Table IV), but without statistical significance $\left(\chi^{2}=1.31, \chi^{2}=2.898\right.$ and $\chi^{2}=1.269$, respectively, $p=0.252, p=0.089$ and $p=0.26$, respectively).

\section{Neuroendoscopy promoted GCS and GOS score}

One week after the operation, the GCS scores were compared between the neuroendoscopy group and the external ventricular drainage + urokinase group. The findings showed that the GCS score in the neuroendoscopy group was significantly higher compared to that in the external ventricular drainage + urokinase group (Table $\mathrm{V}, t=2.189, p=0.028$ ). Meanwhile, the results also demonstrated that 6 months postoperatively, GOS scores were significantly higher in the neuroendoscopy group compared to those in the external ventricular drainage + urokinase group (Table V, $t=2.609, p=0.009$ ).

\section{Neuroendoscopy decreased mRS score}

In this study, patients were followed up for 6 months, and the prognosis was also evaluated using mRS criteria. The mRS score was significantly lower in the neuroendoscopy group compared to that in the external ventricular drainage + urokinase group (Table $\mathrm{V}, t=3.58, p<0.001$ ). This result suggests that the prognosis in the neuroendoscopy group was better than that in the external ventricular drainage + urokinase group.

\section{Discussion}

Neuroendoscopy has been applied in operative treatment of hypertensive intracerebral hemorrhage in our department since 2010. In clinical practice, it has been found that neuroendoscopy also presents a few deficiencies, such as a narrow working channel, limited operating space and difficulty in hemostasis. Meanwhile, the endoscopic field from neuroendoscopy becomes blurred easily, therefore limiting application of neuroendoscopy in treatment of hypertensive intracerebral hemorrhage. Nishihara et al. [15] designed the transparent sheath catheter that can provide a clear surgical field and clearly display the boundary between the hematoma and brain parenchyma. Meanwhile, the angle of the sheath catheter can be adjusted to observe bleeding in the hematoma cavity. However, the angle of the sheath catheter needs to be adjusted according to the requirement of surgery, easily pulling and without damaging surrounding normal tissues and causing iatrogenic injury [15]. Meanwhile, the sheath catheter is fixed, so that the endoscope and instruments have a larger activity space and angle, which could avoid damage of the surrounding normal brain tissues. However, the cortex at the puncture site is also expanded while the hematoma cavity is opened, thereby diminishing the advantage 

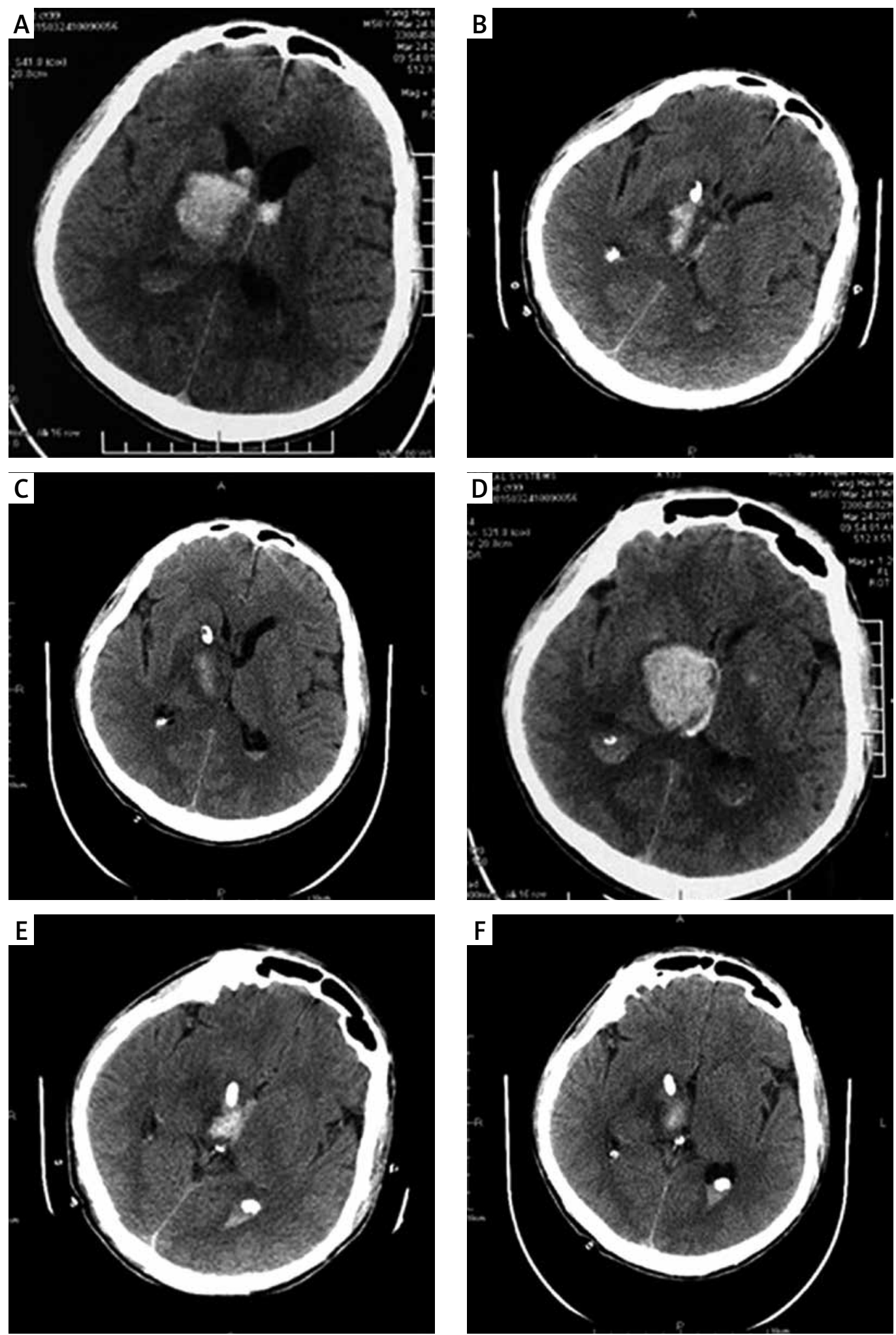

Photo 3. A male patient with thalamic hemorrhage breaking into the ventricle. A - Preoperative CT. $\mathbf{B}-$ CT reexamination is performed immediately after operation, and hematoma is mostly removed. $\mathbf{C}-$ In $\mathrm{CT}$ at $3 \mathrm{~d}$ after operation, hematoma is mostly absorbed, and the drainage tube is withdrawn. $\mathbf{D}$ - Preoperative CT (for another vision). $\mathrm{E}-\mathrm{CT}$ reexamination is performed immediately after operation, and hematoma is mostly removed (for another vision). $\mathrm{F}-\mathrm{In} \mathrm{CT}$ at $3 \mathrm{~d}$ after operation, hematoma is mostly absorbed, and the drainage tube is withdrawn (for another vision) 

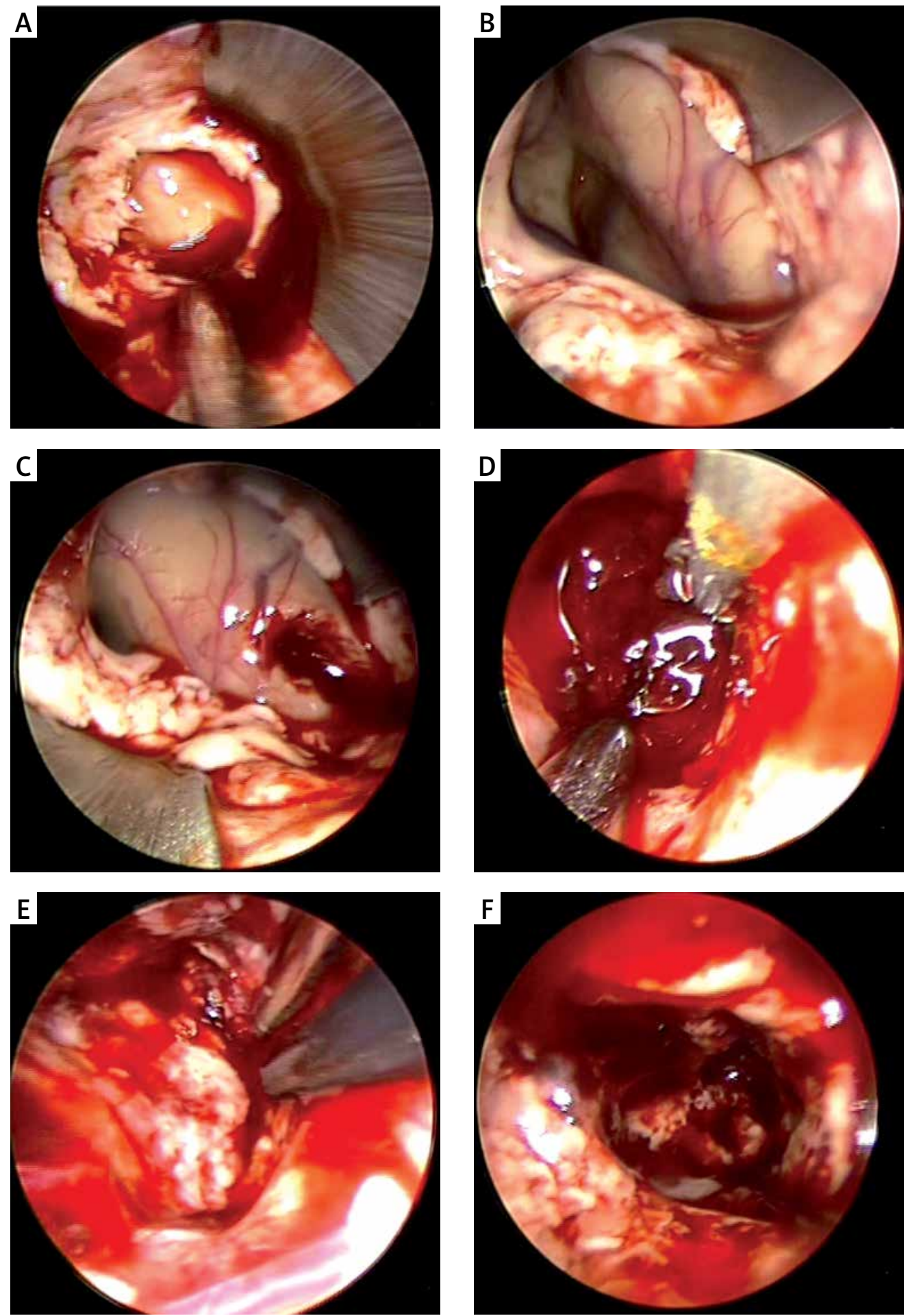

Photo 4. Endoscopic hematoma clearance of thalamic hemorrhage breaking into the ventricle. $\mathbf{A}-$ The ventricle is opened and the bloody cerebrospinal fluid flows out. B - Structure exposed after hematoma clearance in interventricular foramen and third ventricle (arrow). C - The hematoma compresses the ventricle inwards and enters the hematoma cavity through the ventricular wall fistula. D - Hematoma clearance under the endoscope. E - Electro-coagulation of active bleeding under the endoscope (arrow). F- No active bleeding under the endoscope 
Table III. Comparison of hematoma clearance rate in intraventricular hemorrhage

\begin{tabular}{|lccc|}
\hline Group & \multicolumn{3}{c|}{ Hematoma clearance rate } \\
\cline { 2 - 4 } & $90 \%$ & $60-90 \%$ & $<60 \%$ \\
\hline Neuroendoscopy group & 4 & 4 & 2 \\
\hline $\begin{array}{l}\text { External ventricular } \\
\text { drainage + urokinase group }\end{array}$ & 0 & 3 & 6 \\
\hline$\chi^{2}$ & & 6.107 & \\
\hline$P$-value & & 0.047 \\
\hline
\end{tabular}

Table IV. Comparison of postoperative complications of intraventricular hemorrhage

\begin{tabular}{|lccc|}
\hline Group & $\begin{array}{c}\text { Intra- } \\
\text { cranial } \\
\text { infection }\end{array}$ & $\begin{array}{c}\text { Hydro- } \\
\text { cephalus }\end{array}$ & $\begin{array}{c}\text { Pulmo- } \\
\text { nary } \\
\text { infection }\end{array}$ \\
\hline Neuroendoscopy group & 2 & 1 & 3 \\
\hline $\begin{array}{l}\text { External ventricular } \\
\text { drainage + urokinase } \\
\text { group }\end{array}$ & 4 & 4 & 5 \\
\hline$\chi^{2}$ & 1.310 & 2.898 & 1.269 \\
\hline$P$-value & 0.252 & 0.089 & 0.260 \\
\hline
\end{tabular}

Table V. Comparison of prognosis of intraventricular hemorrhage

\begin{tabular}{|lccc|}
\hline Group & $\begin{array}{l}\text { GCS* at } 1 \text { week } \\
\text { after operation }\end{array}$ & $\begin{array}{c}\text { GOS at } 6 \text { months } \\
\text { after operation }\end{array}$ & $\begin{array}{c}\text { mRS at } 6 \text { months } \\
\text { after operation }\end{array}$ \\
\hline Neuroendoscopy group & $12(8-14)$ & $4(3-5)$ & $1(0-3)$ \\
\hline $\begin{array}{l}\text { External ventricular drainage }+ \\
\text { urokinase group }\end{array}$ & $10(6-13)$ & $3(1-4)$ & $4(2-6)$ \\
\hline$t$ & 2.189 & 2.609 & 3.580 \\
\hline$P$-value & 0.028 & 0.009 & $<0.001$ \\
\hline
\end{tabular}

of minimally invasive surgery to a certain extent and increasing surgical trauma [16, 17]. Therefore, a type of modified heron-mouth neuroendoscopic sheath with the sheath of a fixed diameter and an expandable sheath catheter was manufactured in this study. Moreover, the end of the sheath catheter could be dilated through adjusting the sheath handle to increase the surgical field and operating space when necessary.

At the same time, for neuroendoscopy, the cortex injury was not increased due to the limited dilation of the head end. In treatment of hypertensive intracerebral hemorrhage with heron-mouth neuroendoscopic sheath-assisted neuroendoscopy, some surgical experiences and skills have been summarized, such as sulcus separation, sheath locking and double suction technique [18]. In the present study, according to site of hypertensive intracerebral hemorrhage, a 3-4 cm long straight incision was made, drilled and expanded into a bone window $2.0-2.5 \mathrm{~cm}$ in diameter. Then, the dura mater was cut and suspended. The nearest channel to the hematoma cavity was selected using the natural sulcus depth and length. The artery and draining veins in the sulcus were protected during separation. After hematoma needle puncture, the heron-mouth neuroendoscopic sheath was placed slowly and fixed with the snake retractor. Then the sheath core was withdrawn, followed by clearance of intracerebral hematoma under the endoscope. There were no significant differences for age, duration from onset to operation, GCS or Graeb intraventricular hemorrhage score between two groups. Therefore, parameters are comparable between the two groups.

In the past, the tough hematoma was often smashed with forceps, but the efficiency was low and the risk of iatrogenic injury was high in the traditional external ventricular drainage method. The concurrent operation of double instruments can obtain results similar to those of microsurgery, so the double suction technique was adopted for hematoma clearance and hemostasis in this study. The hematoma was separated and removed using two aspirators with $2 \mathrm{~mm}$ diameter through endoscopic working channel and endoscopic external sheath space. The strong suction tube was used to suck out the hematoma, and the weak suction tube was used to separate and pull the hematoma. The hemorrhagic spot was sucked with the weak suction tube in active bleeding, and the mono-polar electro-coagulation contacted the distal suction tube with low current for electro-coagulation hemostasis, which can effectively stop bleeding in most cases. If there were many extradural hematomas or 
extradural hemorrhage of unknown origin, the end of the sheath catheter was dilated by adjusting the sheath handle to open the hematoma cavity. Then, the intracerebral hematoma could be cleaned using double suction technique under direct vision and hemostasis. However, the traditional endoscopy and external ventricular drainage + urokinase method cannot resolve the above problems. In our study, the neuroendoscopy treatment significantly shortened the extubation time compared to that in the external ventricular drainage + urokinase group. However, neuroendoscopy treatment prolonged surgical time, increased the amount of intraoperative bleeding and enlarged the cortex fistula, all of which need to be further improved in the following studies.

Comparing with the external ventricular drainage + urokinase method, the heron-mouth neuroendoscopic sheath provided a more spacious and stable observing and operating environment for endoscopic surgery. Meanwhile, the heron-mouth neuroendoscopic sheath increased the degree of freedom of the surgical operation without increasing the risk of cortex injury. Therefore, the minimally invasive advantage of endoscopic surgery was better retained in this study. The operation using double suction technique can be performed by one person with two hands, significantly speeding up the operation process and effectively improving hematoma clearance efficiency and the hemostatic effect. All of the above are the advantages of neuroendoscopic surgery compared to the external ventricular drainage + urokinase method. The traditional operation of hypertensive intraventricular hemorrhage is the external ventricular drainage combining urokinase dissolution, achieving a definite therapeutic effect [19]. Compared with the external ventricular drainage + urokinase method, the neuroendoscopic surgery also demonstrates obvious advantages, which significantly raised the hematoma clearance rate and improved the long-term prognosis of patients. However, Longatti et al. [20] reported that when treating severe intraventricular hemorrhage using a flexible endoscope, the mortality rate at 1 year was $30 \%$ and the percentage of GOS 3 was $40 \%$ (such as disability), which suggests that the prognosis is not as good as the present results. Therefore, whether the neuroendoscopic surgery presents the reality correctly also needs further discussion in future studies. Meanwhile, the neuroendoscopic surgery group also had lower frequency of postoperative complications, including intracranial infection, hydrocephalus and pulmonary infection, but without statistical significance.

Our results showed that the hematoma clearance rate, ventricular drainage time, intracranial infection and GOS at 1 week/ 6 months after the operation and $\mathrm{mRS}$ at 6 months postoperatively in the neuroendoscopy group were better compared to those in the external ventricular drainage + urokinase group. The surgical time was longer in the neuroendoscopy group than that in the external ventricular drainage + urokinase group. Meanwhile, there was no significant difference for the incidence rate of hydrocephalus between the two groups.

Although this study produced some interesting results, there are also a few limitations. Firstly, the sample size for both the neuroendoscopic surgery group and the external ventricular drainage combining urokinase group was too small. In the next study, we intend to include a larger amount of samples and further investigate the therapeutic efficacy of heron-mouth neuroendoscopic sheath-associated neuroendoscopy on hypertensive intraventricular hemorrhage. Secondly, we have not conducted a typical randomized double-blind controlled investigation. In future, we shall conduct a randomized double-blind controlled study for to demonstrate the advantages of neuroendoscopic surgery more scientifically. Thirdly, this study design did not incorporate a detailed comparison of therapeutic effects between the neuroendoscopic sheath and traditional endoscopy. In the forthcoming investigation, we will further apply neuroendoscopic sheath-associated neuroendoscopy and compare it with traditional endoscopy method. Fourthly, the heron-mouth sheath limits the extent of cortical damage; however, opening of the deep part might be associated with destruction of the periventricular white matter and the subependymal veins. Such destruction might be caused by inexperienced residents. Therefore, the outcome with the heron-mouth sheath could also be affected by the experience of surgeons. Fifthly, the incidences of shunt or endoscopic third ventriculostomy (ETV) in follow-up in both groups were not evaluated in this study. Longatti and Basaldella [9] gave a detailed introduction of their experience with a flexible endoscope for suction of ventricular hematomas, which suggests that neuroendoscopic surgery is a feasible method for rapid clinical improvement. 


\section{Conclusions}

The heron-mouth neuroendoscopic sheath extends the application range of neuroendoscopy in neurosurgery in primary hospitals. There are reasons to believe that minimally invasive surgery combined with navigation technique application in the brain parenchyma and ventricle has broadly applicable prospects.

\section{Conflict of interest}

The authors declare no conflict of interest.

\section{References}

1. Singh R, Baby B, Damodaran N, et al. Design and validation of an open-source, partial task trainer for endonasal Neuroendoscopic skills development: Indian experience. World Neurosurg 2016; 86: 259-69.

2. Hardavella G, Ianovici N. Current trends in minimally invasive neurosurgery: neuroendoscopy. Rev Med Chir Soc Med Nat Lasi 2005; 109: 528-31.

3. Abunimer AM, Abou-Al-Shaar H, Cavallo C, et al. Minimally invasive approaches for the management of intraventricular hemorrhage. J Neurosurg Sci 2018; 62: 734-44.

4. Stachura K, Grzywna E, Krzyzewski RM, et al. Endoscopic biopsy of intra- and paraventricular brain tumors. Videosurgery Miniinv 2019; 14: 107-13.

5. Auer LM, Deinsberger W, Niederkom K, et al. Endoscopic surgerv versus medical treatment for spontaneous inlracerebral hematoma: a mndomized study. J Neurosurg 1989; 70: 530-5.

6. Chen CC, Liu CL, Tung YN, et al. Endoscopic surgery for intraventficular hemorrhage (IVH) caused by thalamic hemorrhage: comparisons of endoscopic surgery and external ventricular drainage (EVD) surgery. World Neurosurg 2011; 75: 264-8.

7. Kuo LT, Chen CM, Li CH, et al. Early endoscope-assisted hematoma evacuation in patients with supratentorial intracerebral hemorrhage: case selection, surgical technique, and long-term results. Neurosurg Focus 2011; 30: E9.

8. Dye JA, Dusick JR, Lee DJ, et al. Frontal bur hole throtLgh an eyebrow incision for image-guided endoscopic evacuation of spontaneous intracerebral hemorrhage. J Neurosurg 2012; 117: 767-73.

9. Longatti P, Basaldella L. Endoscopic management of intracerebral hemorrhage. World Neurosurg 2013; 79: el-7.

10. Basaldella L, Marton E, Fiorindi A, et al. External ventricular drainage alone versus endoscopic surgery for severe intraventricular hemorrhage: a comparative retrospective analysis on outcome and shunt dependency. Neurosurg Focus 2012; 32: E4.

11. Oehalski P, Chivukula S, Shin S, et al. Outcomes after endoscnpic port surgery for spontaneous intracerebral hematomas. J Neural Surg A Cent Eur Neurosurg 2014; 75: 195-205.

12. Mori R, Joki T, Nonaka Y, et al. Parallel insertion endoscopic technique for precise catheter placement in cystic craniopharyngiomas. J Neurol Surg A Cent Eur Neurosurg 2014; 75: 442-6.
13. Feng G, Gao Z, Tian X, et al. Modified two-handed transnasal endoscopic surgery: innovative instrument design and an experimental canine study. Ear Nose Throat J 2017; 96: e24-7.

14. Linzey JR, Wilkinson DA, Nadel JL. Complications in patients undergoing microsurgical clipping of intracranial aneurysms with preexisting ventriculoperitoneal shunts following a cranial procedure. J Stroke Cerebrovasc Dis 2019; 28: 845-9.

15. Nishihara T, Teraoka A, Morita A, et al. A transparent sheath for endoscopic surgery and its application in surgical evacuation of spontaneous intracerebral hematomas. Technical note. J Neurosurg 2000; 92: 1053-5.

16. Waran V, Vairavan N, Sia SF, et al. A new expandable cannula system for endoscopic evacuation of intraparenchymal hemorrhages. J Neurosurg 2009; 111: 1127-30.

17. Zhang HZ, Li P, Yan ZC, et al. Endoscopic evacuation of basal ganglia hemorrhage via keyhole approach using an adjustable cannula in comparison with craniotomy. BioMed Res Int 2014; 2014: 898762.

18. Lu H, Wu CF, Zhu AH. The effect of transparent neuroendoscopic sheath via frontal approach in patients with hypertensive basal ganglia hemorrhage. China Microcycle 2009; 6: 578-9.

19. Chan V, Marro A, Chainey J, et al. Intraventricular atypical teratoid rhabdoid tumour in an adult: a case report and literature review. Folia Neuropathol 2019; 57: 295-300.

20. Longatti P, Fiorindi A, Di Paola F, et al. Coiling and neuroendoscopy: a new perspective in the treatment of intraventricular haemorrhages due to bleeding aneurysms. J Neurol Neurosurg Psychiatry 2006; 77: 1354-8.

Received: 12.04.2020, accepted: 17.07.2020. 